\title{
CORRELATION BETWEEN MAGNESIUM \& INSULIN RESISTANCE IN POLYCYSTIC OVARY SYNDROME (PCOS)
}

\author{
T. Dixit Sweety Saral ${ }^{1}$, Dr. B. Gayathri ${ }^{2 *}$, Dr. Renuka.P ${ }^{3}$, S. Aishwarya ${ }^{4}$, \\ Dr.V. M. Vinodhini ${ }^{5}$, Dr. M. Anuradha ${ }^{6}$ \\ ${ }^{1}$ Post graduate scholar, ${ }^{2}$ Associate professor, ${ }^{3}$ Professor, ${ }^{4}$ Research scholar, ${ }^{5} \mathrm{HOD}$ and \\ Professor, Department of Biochemistry, SRM Medical College Hospital and Research \\ Centre, SRM Institute of Science and Technology, Kattankulathur - 603203, Kanchipuram, \\ Chennai, Tamilnadu, India. \\ ${ }^{6}$ HOD and Professor, Department of Obstetrics and Gynaecology, SRM Medical College \\ Hospital and Research Centre, SRM Institute of Science and Technology, Kattankulathur- \\ 603203, Kanchipuram, Chennai, Tamilnadu, India.
}

*2 Corresponding Author: Dr.B.Gayathri,MD, Associate Professor, Department of Biochemistry, SRM Medical College Hospital and Research Centre, SRM Institute of Science and Technology, Kattankulathur - 603203, Kanchipuram, Chennai, Tamilnadu, India, gayathrb2@srmist.edu.in,

\begin{abstract}
PCOS is a hormonal disorder common among women of reproductive age. Patients with PCOS are known to have a high incidence of insulin resistance. Serum magnesium $(\mathrm{Mg})$ is often associated with insulin resistance among patients with PCOS. This case - control study was conducted at SRM Medical College Hospital and Research Centre, Tamil Nadu, India. Total of 62 participants between the age group of 20-40 years were recruited in the study, in which 31 were healthy controls and 31 were cases with PCOS. FBG, HbAlc, lipid profile, serum magnesium, serum insulin was estimated. Students ' $t$ ' test was done to compare the mean levels between groups. Low serum magnesium, higher levels of insulin and insulin resistance is seen in PCOS compare to non-PCOS group. The correlation of analysed biochemical parameters were done among PCOS group. Negative correlation of mg with insulin, FBS and HOMA-IR was observed. The evidence showing that low serum magnesium is associated with insulin resistance in PCOS individuals.
\end{abstract}

Keywords: PCOS, Magnesium, Insulin resistance, HOMA-IR, Diabetes Mellitus. 


\section{INTRODUCTION}

Polycystic ovary syndrome is a worldwide public health problem. It affected 116 million women $(3.4 \%)$ worldwide in year 2012. Globally, prevalence of PCOS is highly variable, ranging from $2.2 \%$ as high as $26 \%$ [1]. PCOS is a hormonal disorder common affecting woman of reproductive age. Women diagnosed with PCOS are characterised with infrequent or prolonged menstrual periods, excess levels of male hormone (androgen) and ultrasound of ovaries shows numerous small collections of cysts (fluid filled follicles) [2]. The Symptoms of PCOS includes clinically and biochemical hyperandrogenism, infertility hirsutism, acne, alopecia, oligomenorrhoea/amenorrhea, elevated androgen levels [3]. Women with PCOS are at high risk of developing infertility, preeclampsia, early pregnancy loss and endometrial cancer. Furthermore, data suggest that women with PCOS are more likely to develop type 2 diabetes mellitus, dyslipidemia, hypertension, and heart disease as a result of insulin resistance (IR). Obesity which is due to lack of physical activity and family history is also found to be a risk factor of PCOS. Although cysts are not hazardous, they do cause hormonal abnormalities [4].

The homeostatic model assessment of IR (HOMA-IR) is the most widely used approach for IR measurement in scientific investigations because it is easy and non-invasive method with a simple mathematically developed nonlinear equation (HOMA-IR: fasting plasma insulin $(\mu \mathrm{U} / \mathrm{ml}) \times$ fasting plasma glucose $(\mathrm{mg} / \mathrm{dl}) / 405$ (normalising factor)). The homeostasis model assessment (HOMA) calculates IR and pancreatic -cell function from basal glucose and insulin (or C-peptide) levels [5].

Magnesium acts as a second messenger in insulin action. Hence, magnesium is required for appropriate glucose utilisation and insulin signalling. Studies have shown that serum magnesium levels have been linked to poor glucose tolerance and IR with an increased risk of developing type 2 diabetes mellitus [4]. Hence, this case-control study was planned to estimate the levels of serum magnesium, insulin and to calculate HOMO-IR among patients with PCOS.

\section{MATERIALS \& METHODS}

This case-control study recruited 62 patients in which 31 were in a case group diagnosed with polycystic ovary syndrome using ultrasound and 31 were in healthy control. Patients attending outpatient department of Obstetrics and Gynaecology diagnosed with PCOS.

PCOS subjects were selected based on Rotterdam criteria: Out of the three criteria, two are required for diagnosis: [6]

- Hyperandrogenism

- Clinical (hirsutism or less commonly male pattern alopecia) or

- Biochemical (raised free androgen index or free testosterone).

- Oligo/anovulation.

- Polycystic ovaries on ultrasound.

Subjects were excluded from the study if they ultrasound formed case group and healthy female volunteers attending the master health check-up programme at SRM Medical College 
Hospital \& Research Centre, Kattankulathur, Kanchipuram, Chennai, Tamil Nadu, India formed control group.

PCOS is defined with irregular periods, infertility, presence of polycystic ovaries on ultrasound scans. Participants between the age group of 20-40 years diagnosed with Polycystic Ovary Syndrome (PCOS) using ultrasound were included in the study. Patients with the history of using oral contraceptives and with the clinical conditions of diabetes mellitus, thyroid disorders, renal diseases, cushing's syndrome, hypertension and cardiovascular diseases were excluded from the study. Pregnant or lactating women and female in the age group of $<20 \&>40$ years were also excluded.

After getting informed written consent from all subjects, under aseptic precaution, $5 \mathrm{ml}$ of venous blood sample was collected from anticubital vein after overnight fasting of 10-12 hours in appropriate vacutainer. After centrifugation at $3000 \mathrm{rpm}$ for 10 minutes, serum was separated from whole blood sample and was subsequently analysed for serum magnesium, total cholesterol, triglyceride, LDL-C and HDL-C in Beckman Coulter Auto-analyser (AU480). Serum insulin was analysed in VITROS Eci immunoanalyzer, plasma sample was used for estimation of fasting glucose in Beckman Coulter Auto - analyser (AU-480) and the whole blood were used for estimation of HbA1c in Bio RAD D10.

The study protocol was performed in accordance with the approval of the Institutional ethical committee (ECN: 2866/IEC/2021).

\section{Statistical analysis}

Analysis of variables was performed by using SPSS software 25.0 version. Data were statistically described in term of mean and standard deviation (SD). Comparison between the two groups was done using the student ' $t$ ' test. The correlation between variables was assessed using Pearson's correlation equation. $\mathrm{p}$ value $<0.05$ was considered to be statistically significant.

\section{RESULTS}

A total 62 subject were studied in which 31 subjects diagnosed with polycystic ovary syndrome (PCOS) were in case group and 31 healthy subjects were in control group. The mean age of controls and cases were $23.77 \pm 3.42$ and $25.32 \pm 3.87$ years respectively. The data were distributed according to the age group as 20-25 years, 26-30 years, 31-35 years and 36-40 years (Table 1 ).

Table 1. Distribution of Subjects according to age

\begin{tabular}{|c|c|c|c|c|}
\hline AGE & \multicolumn{2}{|c|}{ CONTROL } & \multicolumn{2}{c|}{ CASE } \\
\hline $20-25$ & 28 & $90 \%$ & 18 & $58 \%$ \\
\hline $26-30$ & 2 & $7 \%$ & 12 & $39 \%$ \\
\hline $31-35$ & 0 & $0 \%$ & 0 & $0 \%$ \\
\hline $35-40$ & 1 & $3 \%$ & 1 & $3 \%$ \\
\hline Total & 31 & $100 \%$ & 31 & $100 \%$ \\
\hline
\end{tabular}




\begin{tabular}{|l|l|l|}
\hline Mean \pm SD & $23.77 \pm 3.42$ & $25.32 \pm 3.87$ \\
\hline
\end{tabular}

The mean BMI $\left(\mathrm{Kg} / \mathrm{m}^{2}\right)$ for control $23.07 \pm 1.51$ and case $28.26 \pm 6.33$ were calculated and found to be significant between groups. The data were distributed based on BMI as normal, overweight and obese patients according to the WHO standards and found that $48 \%$ of PCOS individual were overweight (Table 2).

Table 2. Distribution of Subjects according to BMI

\begin{tabular}{|c|c|c|c|c|c|}
\hline BMI $\left(\mathrm{Kg} / \mathrm{m}^{2}\right)$ & \multicolumn{2}{|c|}{ Control } & \multicolumn{2}{|c|}{ Case } & p value \\
\hline Normal & 30 & $97 \%$ & 8 & $26 \%$ & \multirow{5}{*}{$<0.0001^{* * *}$} \\
\hline Overweight & 1 & $3 \%$ & 15 & $48 \%$ & \\
\hline Obese & 0 & $0 \%$ & 8 & $26 \%$ & \\
\hline Total & 31 & $100 \%$ & 31 & $100 \%$ & \\
\hline Mean \pm SD & \multicolumn{2}{|c|}{$23.07 \pm 1.51$} & \multicolumn{2}{|c|}{$28.26 \pm 6.33$} & \\
\hline
\end{tabular}

The mean values of Magnesium, Insulin, HOMA-IR, fasting blood glucose, HbA1c, Total Cholesterol, Triglyceride, HDL-C, LDL-C and VLDL-C were compared between the PCOS patients and healthy subjects with the help of student's t-test. The mean levels of Insulin, HOMA-IR (Figure.1), fasting blood glucose, Total Cholesterol, Triglyceride, HDL-C, LDL$\mathrm{C}$ and VLDL-C were significantly higher in case group compare to control group. The mean level of Mg was significantly decreased in cases compare to controls (table 3).

Table 3. Comparison of Mean \pm SD of the Measured Biochemical Parameters between the PCOS Patients (Case) and Healthy Subjects (Control).

\begin{tabular}{|c|c|c|c|}
\hline PARAMETER & $\begin{array}{c}\text { CASE } \\
\text { Mean } \pm \text { SD }\end{array}$ & $\begin{array}{c}\text { CONTROL } \\
\text { Mean } \pm \text { SD }\end{array}$ & p value \\
\hline Mg $(\mathbf{m g} / \mathbf{d l})$ & $1.90 \pm 0.15$ & $2.28 \pm 0.37$ & $<0.0001^{* * *}$ \\
\hline Insulin( $\boldsymbol{\mu U} / \mathbf{m l})$ & $19.18 \pm 8.95$ & $9.95 \pm 4.87$ & $<0.0001^{* * *}$ \\
\hline HOMA-IR & $5.09 \pm 2.42$ & $2.36 \pm 1.19$ & $<0.0001$ \\
\hline Fasting blood glucose (mg/dl) & $107.26 \pm 6.04$ & $95.39 \pm 4.49$ & $<0.0001^{* * *}$ \\
\hline HbA1c(\%) & $5.25 \pm 0.31$ & $5.38 \pm 0.29$ & $0.36^{\mathrm{NS}}$ \\
\hline T-Cholesterol(mg/dl) & $175.16 \pm 28.55$ & $152.71 \pm 17.89$ & $0.0005^{* * *}$ \\
\hline Triglycerides(mg/dl) & $96.90 \pm 44.75$ & $74.10 \pm 25.74$ & $0.0168^{*}$ \\
\hline HDL-C(mg/dl) & $46.61 \pm 10.38$ & $48.87 \pm 7.42$ & $0.32^{\mathrm{NS}}$ \\
\hline LDL-C(mg/dl) & $126.19 \pm 26.29$ & $105.84 \pm 15.71$ & $0.0005^{* * *}$ \\
\hline VLDL & $19.39 \pm 9.00$ & $15.23 \pm 6.26$ & $0.0388^{*}$ \\
\hline
\end{tabular}

Mg: Magnesium; HOMA-IR: Homeostatic Model Assessment of Insulin Resistance; FBG: Fasting blood glucose; HbA1c: Glycated haemoglobin; Tc: Total cholesterol; TG: Triglycerides; HDL-c: High density lipoprotein cholesterol; LDL-c: Low density lipoprotein cholesterol and VLDL-c Very low-density lipoprotein cholesterol. *significant $(\mathrm{p}<0.05)$. 
Figure 1. Comparing the Mean Levels of HOMA-IR in Case and Control (Bar Graph)

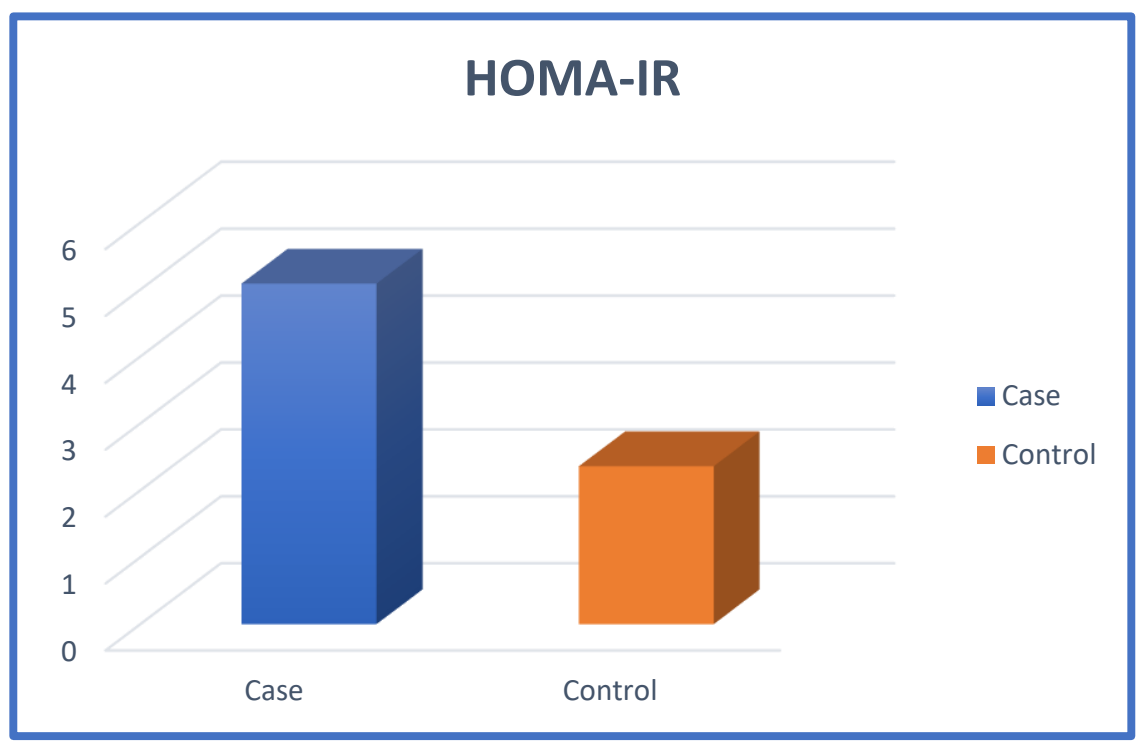

Pearson' s correlation was done for Mg with Insulin, HOMA-IR, TC, LDL-c and HDL-c. A significant positive correlation was observed between $\mathrm{Mg}$ and total cholesterol. Insulin and HOMA-IR was negatively correlated with Mg (Table 4).

Table 4. Pearson's correlation coefficient between Magnesium and other laboratory parameters in PCOS patients.

\begin{tabular}{|c|c|c|}
\hline $\begin{array}{c}\text { Correlation of magnesium } \\
\text { with }\end{array}$ & r VALUE & p- value \\
\hline Insulin $(\mu \mathrm{U} / \mathrm{ml})$ & -0.14501 & 0.43 \\
\hline HOMA-IR $(\mathrm{mg} / \mathrm{dl})$ & -0.14198 & 0.447 \\
\hline $\mathrm{TC}(\mathrm{mg} / \mathrm{dl})$ & 0.3689 & $0.041^{*}$ \\
\hline LDL -c $(\mathrm{mg} / \mathrm{dl})$ & 0.2550 & 0.166 \\
\hline HDL-c $(\mathrm{mg} / \mathrm{dl})$ & 0.2608 & 0.156 \\
\hline
\end{tabular}

TC: Total cholesterol; HOMA-IR: Homeostatic Model Assessment of Insulin Resistance; LDL-C: Low density lipoprotein cholesterol; HDL-C: High density lipoprotein cholesterol; *significant $(\mathrm{p}<0.05)$.

Pearson's correlation of insulin with total cholesterol and LDL-c was positive and with HDLc was negative (Table 5).

Table 5. Pearson's correlation coefficient Insulin and other laboratory parameters in PCOS patients.

\begin{tabular}{|c|c|c|}
\hline $\begin{array}{c}\text { Correlation of Insulin } \\
\text { with }\end{array}$ & r- Value & p-Value \\
\hline $\mathrm{TC}(\mathrm{mg} / \mathrm{dl})$ & 0.1191 & 0.523 \\
\hline LDL -c $(\mathrm{mg} / \mathrm{dl})$ & 0.2457 & 0.183 \\
\hline HDL-c $(\mathrm{mg} / \mathrm{dl})$ & -0.2700 & 0.142 \\
\hline
\end{tabular}

TC: total cholesterol; LDL-C: low density lipoprotein cholesterol; HDL-C: high density lipoprotein cholesterol; *significant (p<0.05). 


\section{DISCUSSION}

A total of 31 patients with a confirmed diagnosis of PCOS and 31 healthy controls were chosen to investigate the levels of biochemical parameters namely fasting glucose, magnesium, total cholesterol, triglycerides, HDL-C, LDL-C, and VLDL-C.

Compared to the control group, PCOS women had a considerably higher BMI. Obesity increases the risk of PCOS, according to the findings of the current study, which are comparable to the studies of Saxena et al, Blasco et al, Sanchez N et al, Majumdar et al, and Yildiz et al [11].

V.M. Vinodhini et al., (2012) found no statistically significant differences in mean fasting blood glucose concentrations between PCOS patients and healthy controls [7], However Azevedo MF et al., (2011) found statistically significant differences in fasting blood glucose levels in PCOS women [8]. Our findings matched those of Azevedo MF et al research.

According to second study Kauffman RP et al. (2011) found that no difference between PCOS and normal women for their serum magnesium concentration levels and reported that magnesium levels did not correlate with age, BMI, glycaemic levels, blood pressure and lipid levels in women with PCOS. But, the present study showed decreased magnesium levels in PCOS patients as compared to controls and a weekly negative correlation between glucose, insulin and HOMA-IR with magnesium levels [9]. The population in this study was too small to do a good interpretation on their results.

The lipid profile of PCOS patients was also measured and compared to that of control groups. Olivier Valkenburg et al. (2008) found that PCOS patients had higher levels of total cholesterol, triglycerides, and LDL-C than controls. HDL-C levels in women with PCOS, on the other hand, were considerably lower [10]. Our result was also consistent with the above study. We also found a non-significant negative correlation between glucose and HDL-C and significant positive correlation between glucose and total cholesterol, triglycerides, LDL-C. In a study by maltezos et al, provides evidence that magnesium insufficiency is not linked to IR in PCOS and study by Kaufmann et al, concluded that there was no association between serum $\mathrm{Mg}$ concentrations and insulin secretion or IR indices [9]. In our study Mg status suggest, there is a relation between serum $\mathrm{Mg}$ concentrations and IR among women with PCOS.

Obese PCOS women are commonly thought to be insulin-resistant [13]. In comparison to obese PCOS women in this study overweight PCOS women exhibit higher insulin levels and more prominent IR [13].

Kanafchian et al, found no statistically significant differences in serum Mg levels between the groups, but did detect a negative association between $\mathrm{Mg}$ with Fbs and HOMA-IR in women with Insulin resistance and PCOS [12]. Our finding also matched those of kanafchian et.al research. 


\section{CONCLUSION}

In this case - control study low serum magnesium levels were observed among PCOS. Higher BMI significantly increase fasting blood glucose, total cholesterol, TGL, LDL-C and VLDL$\mathrm{C}$ and low HDL-C seen in case group compare to non-PCOS group. Higher levels of insulin are seen among PCOS group compare to non-PCOS group. Hence higher insulin resistance is observed among PCOS individual. This evidence shows that low serum magnesium levels among PCOS individuals is associated with insulin resistance.

Moreover, the true cause of disease is unknown. Although uncontrolled steroidogenesis, IR, genetics, and oxidative stress are likely to play a role, more research is needed to better understand the disease in order to prevent comorbidities and treat PCOS. Longer-term research examining insulin resistance in PCOS and PCOS phenotypes are required.

\section{ACKNOWLEDGEMENT}

We want to thank the women who participated in this study. The authors would like to express their gratitude to SRM MHC \& RC, Department of Biochemistry for providing laboratory facilities, and department of Obstetrics and Gynaecology for sample collection and cooperation.

\section{Conflict of Interest:}

The authors declare that they have no conflicts of interest.

\section{REFERENCE:}

1. World Health Organization. Global status report on noncommunicable diseases 2012 description of the global burden of NCDs, their risk factors and determinants 2011.

2. Anna Rajska, Magdalena Buszewska-Forajta, Dominik Racho 'n and Michat Jan Markuszewski ,* Metabolomic Insight into Polycystic Ovary Syndrome. International Journal of Molecular Sciences 2020.

3. N.Swetha, R. Vyshnavi, P. Modagan, Balaji Rajagopalan. A correlative study of biochemical parameters in polycystic ovarian syndrome Int J Biol Med Res. 2013; 4(2): 3148- 3154

4. Mahesh Gupta, Daneshwar Singh, Manju Toppo, Angelin Priya, Soumitra Sethia, Preeti Gupta. A cross sectional study of polycystic ovarian syndrome among young women in Bhopal, Central India. International Journal of Community Medicine and Public Health 2018 Jan;5(1):95-100.

5. Faranak Sharifi, Sahar Mazloomi, Reza hajihosseini. Serum magnesium concentrations in polycystic ovary syndrome and its association with insulin resistance Gynecological Endocrinology, 2012; 28(1): 7-11

6. Renuka.P, Shakthya, Vinodhini VM. Study of Glycated hemoglobin levels in Polycystic ovary syndrome.Asian Journal of Pharmaceutical and Clinical Research,2018 Jan11(5):191-193.

7. V.M.Vinothini, V. Devisri, W.Ebenezer William, M.Muthulakshmi, Anjalakshi chandrasekar and S.Gnanasambandam. High Sensitive C Reactive Protein and Apolipoprotein B levels in 
Polycystic ovary syndrome. International Journal of Pharma and Bio Sciences (2012); 3(2):719-724

8. Azevedo MF, Costa EC, Oliveira AI, Silva IB et al. Elevated blood pressure in women with Polycystic ovary syndrome. Prevalence and associated risk factors. Rev. Bres. Ginecol Obstet. (2011);33(1):31-36

9. Kauffman RP, Tullar PE, Nipp RD, Castracane VD. Serum magnesium concentrations and metabolic variables in polycystic ovary syndrome. Acta Obstet Gynecol Scand 2011;90:452458.

10. Olivier Valkenburg, Regine P.M, Steegers-Theunissen, Huberdina P.M. Smedts, Geesje M. Dallinga-Thie, Bart C.J.M. Fauser. A more atherogenic serum lipoprotein profile is present in women with polycystic ovary syndrome: A case-control study. J ClinEndocrinolMetab (2008); 93(2):470-476.

11. Muneyyirci-Delale O, Nacharaju VL, Dalloul M, Jalou S, Rahman M, Altura BM, Altura BT. Divalent cations in women with PCOS: implications for cardiovascular disease. Gynecol Endocrinol 2001;15:198-201.

12. Kanafchian M, Esmaeilzadeh S, Mahjoub S, Rahsepar M, Ghasemi M. Status of Serum Copper, Magnesium, and Total Antioxidant Capacity in Patients with Polycystic Ovary Syndrome. Biol Trace Elem Res. 2019.

13. Arya, B.K.; Haq, A.U.; Chaudhury, K. Oocyte quality reflected by follicular fluid analysis in poly cystic ovary syndrome (PCOS): A hypothesis based on intermediates of energy metabolism. Med. Hypotheses 2012, 78, 475-478. 